\title{
Supporting Multidisciplinary Teams and Early Design Stages Using Storyboards
}

\author{
Mieke Haesen, Jan Meskens, Kris Luyten, and Karin Coninx \\ Hasselt University - tUL - IBBT, \\ Expertise Centre for Digital Media, \\ Wetenschapspark 2, B-3590 Diepenbeek, Belgium \\ \{mieke.haesen, jan.meskens, kris.luyten, karin.coninx\}@uhasselt.be
}

\begin{abstract}
Current tools for multidisciplinary teams in user-centered software engineering (UCSE) provide little support for the different approaches of the various disciplines in the project team. Although multidisciplinary teams are getting more and more involved in UCSE projects, an efficient approach to communicate clearly and to pass results of a user needs analysis to other team members without loss of information is still missing. Based on previous experiences, we propose storyboards as a key component in such tools. Storyboards contain sketched information of users, activities, devices and the context of a future application. The comprehensible and intuitive notation and accompanying tool support presented in this paper will enhance communication and efficiency within the multidisciplinary team during UCSE projects.
\end{abstract}

\section{Introduction}

When combining HCI techniques and software engineering principles in user-centered software engineering (UCSE), the biggest challenge is the communication within a multidisciplinary team including the end users. MuiCSer, a framework for Multidisciplinary user-centered Software Engineering processes, focuses on the benefits of both disciplines, and was introduced to investigate the features and shortcomings of current UCSE models and tools [1]. One missing link in most user centered processes is a tool to progress from informal design artefacts (e.g. scenario) toward more structured design artefacts (e.g. task model). Most tools and techniques require specific knowledge about specialized notations or models, thus exclude most team members to be involved. Furthermore, functional information may be missing in informal design artefacts while structured design artefacts may not always contain all non-functional information. We propose the usage of storyboards as a comprehensible artefact related to features of graphical user interface design tools to overcome these shortcomings. 
In summary, the main contributions in this paper are:

- a novel user-centered design approach that uses storyboards as a common language in a multidisciplinary team;

- tool support for creating and editing storyboards in order to bridge the gap between the early stages of the UCSE process and the user interface design. This tool supports the connection between storyboards and artefacts created later in the process.

\section{Related work}

User-centered processes recommend combining non-functional as well as functional requirements by involving a multidisciplinary team [2]. The early design stages of usercentered design (UCD) include a user needs analysis and generally result in several artefacts such as usability requirements [3], scenarios [4] and personas [5] describing the user needs. These artefacts are written in a narrative style and are usually created by interaction designers. Similar artefacts are used in software engineering and agile development [6] (e.g. essential use cases, scenarios, story cards, user stories).

Although several disciplines provide notations to describe user needs, the notations are not always comprehensible for all members of a multidisciplinary team. Lindgaard et al. [7] address the difficulties in presenting user needs for requirements engineering.

Earlier studies describe the needs of interaction designers in a multidisciplinary team. Brown et al. [8] conducted an ethnographic study to investigate the collaboration between user interaction designers and developers. The study describes the benefits of stories and sketches in the early stages of user-centered approaches and emphasizes the power of combining both. Assembling stories and sketches is a powerful technique to reveal errors, and to consider temporal and contextual information.

Arecent study of Myers et al. [9] reports that designers are experiencing difficulties when designing the behavior of user interfaces. While prototyping the appearance of user interfaces is straightforward, designing and communicating the behavior is an ongoing process. Furthermore, the survey revealed designers frequently use sketches and storyboards.

Currently little tool support is available for storyboarding in multidisciplinary teams. Demais [10] and IBM Rational Requirements Composer ${ }^{1}$ focus on storyboards in the design process of multimedia applications while Denim [11] and Highlight [12] feature storyboards for web applications. All these tools are developed to describe the behavior of software or web applications and support a first walkthrough of the future system or website. The storyboards created using these tools contain mock-ups of UI designs and their relationships, and thus are designed after the requirements gathering of a future application.

\footnotetext{
${ }^{1}$ http://www.ibm.com/developerworks/rational/library/08/1118_zhuo, last visited 6 January 2009
} 
The ActivityDesigner [13] tool allows storyboarding at the early stages of design. In this tool, designers can extract activities from concrete scenarios making it possible to include rich contextual information about everyday lives as scenes. Based on the scenes, higher level structures and prototypes can be created. The tool we present in this paper also provides the possibility to build storyboards during the gathering of requirements in order to facilitate the creation of artefacts at later stages.

\section{Storyboards}

In a UCSE process, a report of a user needs analysis, scenarios and personas are presented to the entire team after conducting the first user studies. Structuring artefacts that are written in a narrative style is a complex though important process. All artefacts created at later stages need to be consistent with these first results. Unfortunately little tool support is available for the first transition in UCSE processes. This implies that the entire team needs to verify consistency among the informal results of a user needs analysis and artefacts created later in the process. A good understanding within the multidisciplinary team at this point can be crucial for the resulting user experience. We investigate how storyboards can be used in UCSE processes by a multidisciplinary team.

\subsection{Users, activities, devices, context}

The professional use of storyboards originates from the film-industry and is getting introduced in several disciplines such as advertisement and product design [14]. In UCSE a storyboard can have several meanings. Storyboards can depict manual steps, users interacting with a product, screen mockups of a new work practice or the link with the system behind-the-scenes [6]. The focus on visual information renders it highly comprehensible for any member of the team, independent of their background or role in the team [14] [15].

In the context of our research, we want to define storyboards as sketches of real life situations, depicting users carrying out several activities by using devices in a certain context. An example of a simple storyboard is presented in the center of Fig. 1. Since storyboards contain a lot of information about the future use of an application, they can be used to provide a link between a user needs analysis and requirements gathering, containing functional as well as non-functional requirements. Furthermore, the natural style of presenting the use of a future system implies this artefact is very comprehensible for all team members including end users.

Since scenes of a storyboard contain contextual information, they are suitable for the specification of context-aware applications. This contextual information has to be taken into account during the entire development process, thus storyboards can contribute to the evaluation, verification and validation of several stages. 


\subsection{Bridging the early stages of UCSE processes}

The creation of storyboards happens at the early stages of a UCSE process, after the creation of scenarios and personas. An example storyboard and the interrelationship between a storyboard and other artefacts are presented in Fig. 1. A storyboard is built by splitting up the scenario into scenes and presenting the scenes as sketches depicting users interacting with the future system. Connecting scenes of a storyboard, structures the narrative information of the scenario. The understandability of storyboards increases the amount of team members that can collaborate during this phase. Even end-users can be involved to create or evaluate storyboards.

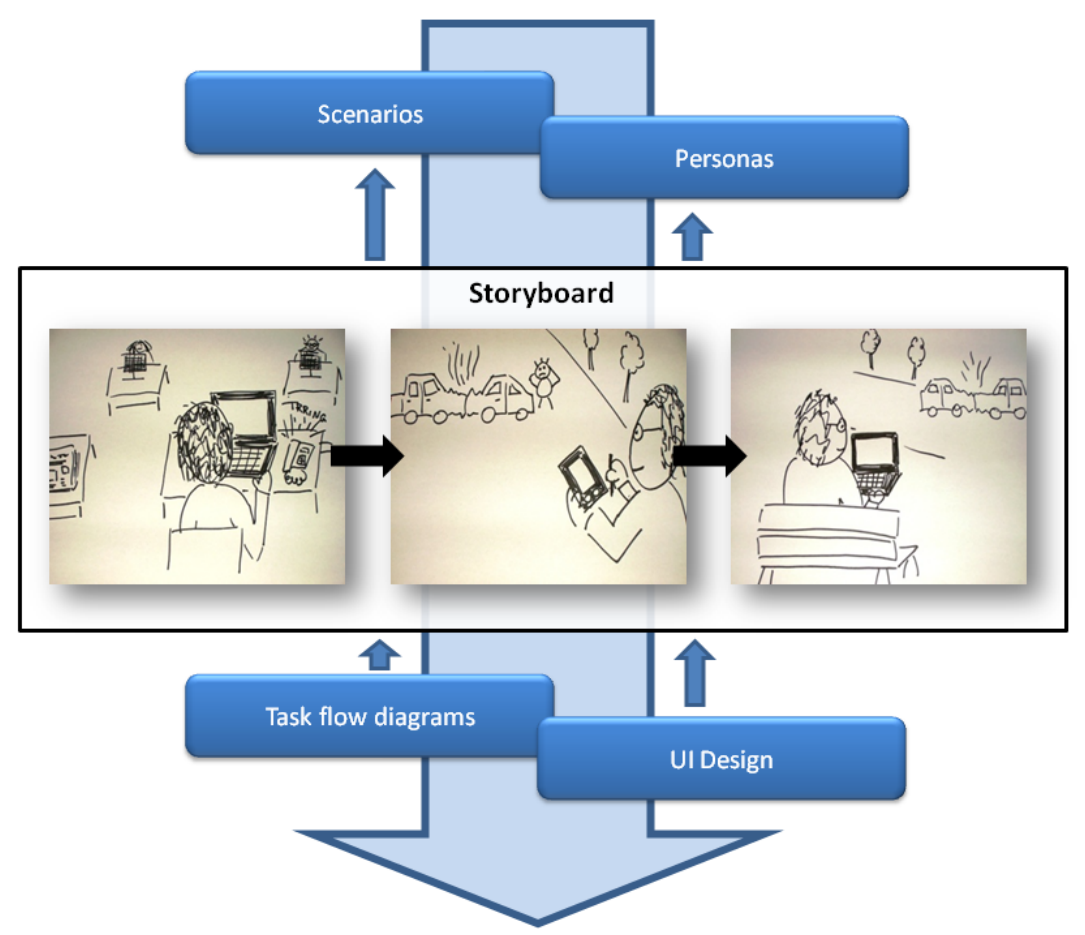

Fig. 1. A storyboard and its interrelationship with other artefacts in the development process. Situations and devices in the scenes are extracted from scenarios, while the user information is extracted from the scenarios as well as the personas. The storyboard is used as input for the creation of task flow diagrams and the UI designs. 
Once all scenes are added to the storyboard, personas and devices can be highlighted in each individual scene. This enriches the information contained by the storyboard and can be used to make the transition to other artefacts. Task flow diagrams, presenting user actions and processes to complete a task, can be produced based on the information in and the connections between the scenes of the storyboard. At a later stage of the development process, the storyboard can guide the UI design and development. By carefully considering the situation of each scene, designers and developers build an application corresponding to context, requirements and constraints contained by the storyboard. Interaction designers can use a storyboard to verify that the UI designs take into account all requirements.

A storyboard also contributes to the preparation of the usability tests. Using storyboards in UCSE processes increases the visibility of the project. New team members for instance, can explore the requirements of the project at a glance by looking at the storyboard.

\section{Tool support for storyboards}

As stated above, storyboards contribute to the development process of software applications in multidisciplinary teams. When suitable tool support is available for all team members, storyboards become more powerful and the visibility and traceability of a project increase.

A literature survey [1] showed there is a need for tools that support UCSE processes in the early stages of design. Since storyboards are created during the requirements gathering, storyboarding tools can partly cover transitions between the early stages of UCSE. Furthermore, storyboards are a very suitable artefact to specify the use of contextaware applications, thus we decided to integrate the tool support for storyboards in the Gummy [16] GUI builder tool. Gummy supports graphical design of multi-device and context-aware user interfaces. To enable this, Gummy automatically adapts its workspace according to the considered target platform and thus allows designers to create user interfaces for a wide range of devices without having to change their work practices. The inclusion of storyboards during this design stage better describes the context of a user interface and provides a more convenient way to describe the intended context-of-use. This way, the storyboards provide guidelines for the design of the UI.

In the storyboarding extension of Gummy, a team member, e.g. an interaction designer starts the creation of the storyboard by loading a scenario into the workspace. Following, a sequence in the scenario can be selected and consequently, a new scene can be created. The sequence of the scenario is automatically added to the scene as a description, while the interaction designer can load an image and add a title. The image of the scene can be a photo of the user observations or a scanned sketch, which encourages designers to sketch in a creative and informal way [11]. A screenshot of the storyboarding tool is shown in Fig. 2. 
For each scene in a storyboard, team members can add annotations and point out the personas and devices. When the specifications of a device (e.g. screen size) are included in the scenes, this information can be considered when the workspace for the UI design is loaded. The contextual information of the scenes (e.g. sketches presenting the environment or courses of communication) can be used as guidelines for the UI design without obstructing the creativity of UI designers.

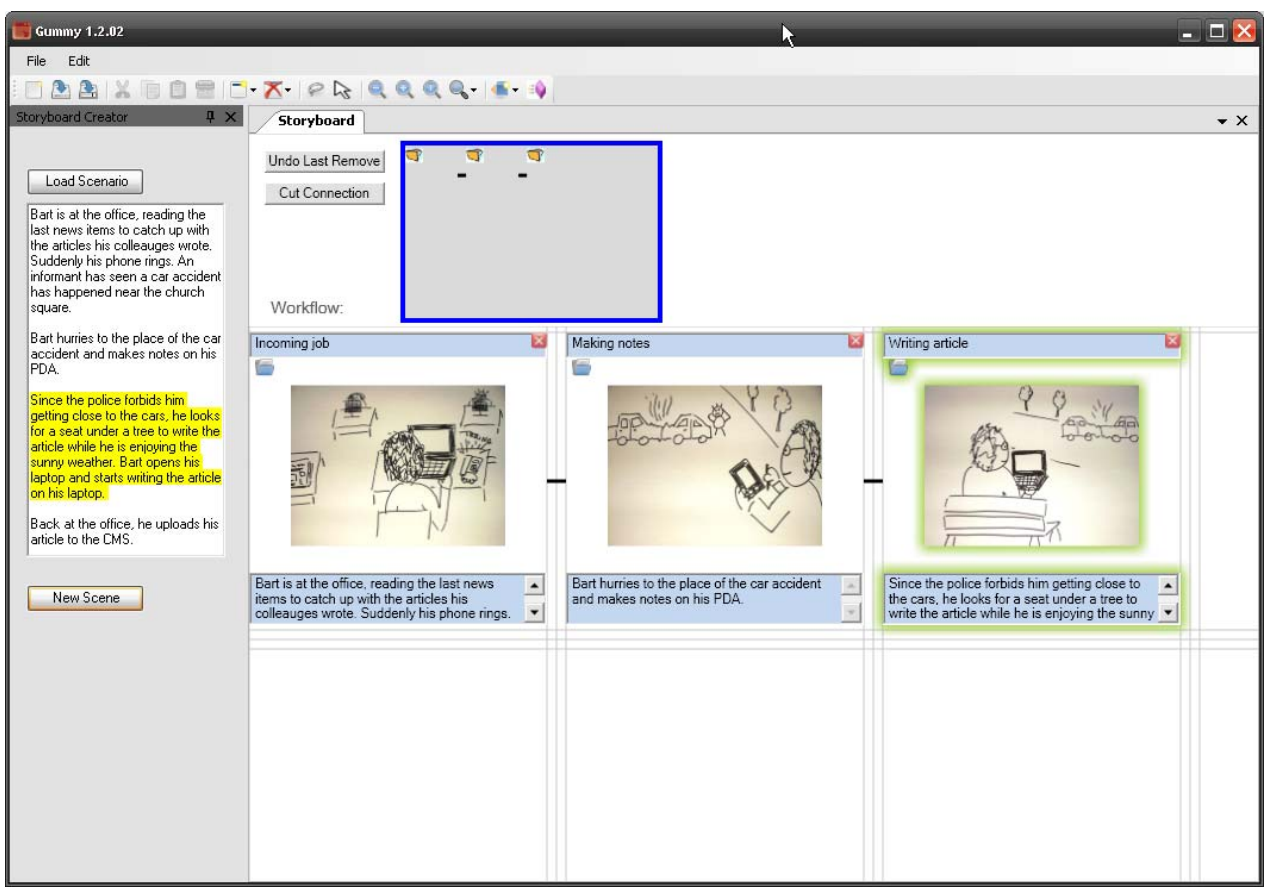

Fig. 2. A screenshot of the storyboarding extension in Gummy. Scenarios can be loaded on the left panel. For a selected sequence in the scenario a new scene can be created. A scene can contain sketches of users interacting with the future system, a title and a description.

By extending existing tool support, the visibility and traceability of UCSE processes can be enhanced. The storyboard extension makes it possible to include the results of the first UCSE stages (user needs analysis) and helps to process and structure these narrative artefacts. Furthermore, a visualization of a scenario by scenes makes it possible to see the usability requirements at a glance, which improves the communication and efficiency in the project team. 


\section{Ongoing and future work}

Storyboards are implicitly used in different ways by multidisciplinary teams. This partly explains the many interpretations of storyboards and reveals the challenges in developing a storyboarding tool for multidisciplinary teams. In ongoing work we are carrying out a survey considering the roles in a multidisciplinary team and the tools used by members of a team. Observations and interviews are organized to investigate current practices of multidisciplinary teams in industry. Furthermore, storyboards as defined in this paper are introduced in a multidisciplinary project team and the storyboarding tool will be evaluated during several iterations.

Based on the findings of these studies, we will fine-tune the features of storyboards and the relationships between storyboards and other artefacts. As the current version of the storyboarding tool is intended for individual use, the user studies may also reveal some expectations of teams regarding a distributed and a collaborative version of the tool. Furthermore, contextual information and platform specifications can be extracted from the scenes in a storyboard to guide design of UIs in the Gummy GUI-builder.

\section{Conclusion}

In this paper we described how storyboards can contribute to UCSE. By sketching users interacting with a future application, pointing out devices and adding annotations in the early stages of a UCSE project, these storyboards contain functional and nonfunctional requirements.

Storyboards can contain rich contextual information and are based on an intuitive notation providing more structure than narrative scenarios of use. We integrated tool support for the creation and the use of storyboards in the Gummy multi-device GUI builder. Ongoing and future studies are being carried out to examine the approach of multidisciplinary teams in industry and to adapt the storyboarding tool according to current practices.

This new level of tool support can simplify the creation of artefacts at later stages of a development process and improves the communication within a multidisciplinary team. The comprehensibility of storyboards allows non-technical team members to be involved in the first activities of model-based UI development. Consequently, the loss of information after a user needs analysis will decrease while the visibility and traceability of a project increase. Storyboards are a common language in multidisciplinary teams, which contributes to the user experience of the final user interface. 
Acknowledgements. Part of the research at EDM is funded by EFRO (European Fund for Regional Development) and the Flemish Government. The MuiCSer process framework and the Gummy tool, including the storyboarding extension, are based on our experiences in the IWT project AMASS++ (IWT 060051).

\section{References}

[1] Mieke Haesen, Karin Coninx, Jan Van den Bergh, and Kris Luyten. MuiCSer: A Process Framework for Multi-Disciplinary User-Centered Software Engineering processes. In Proceedings of Human-Centred Software Engineering, pages 150 - 165, September 2008.

[2] International Standards Organization. ISO 13407. Human Centred Design Process for Interactive Systems. Geneva, Swiss, 1999.

[3] D. Redmond-Pyle and A. Moore. Graphical User Interface Design and Evaluation. Prentice Hall, London, 1995.

[4] John M. Carroll. Making use : scenario-based design of human-computer interactions. MIT Press, Cambridge, Mass. [u.a.], 2000.

[5] John Pruitt and Tamara Adlin. The Persona Lifecycle : Keeping People in Mind Throughout Product Design. Morgan Kaufmann, 2006.

[6] Karen Holtzblatt, Jessamyn B. Wendell, and Shelley Wood. Rapid Contextual Design: A How-to Guide to Key Techniques for User-Centered Design (Interactive Technologies). Morgan Kaufmann, December 2004.

[7] Gitte Lindgaard, Richard Dillon, Patricia Trbovich, Rachel White, Gary Fernandes, Sonny Lundahl, and Anu Pinnamaneni. User needs analysis and requirements engineering: Theory and practice. Interact. Comput., 18(1):47-70, 2006.

[8] Judith Brown, Gitte Lindgaard, and Robert Biddle. Stories, Sketches, and Lists: Developers and Interaction Designers Interacting Through Artefacts. In Proceedings of Agile 2008, pages 39 - 50, 2008.

[9] Brad A. Myers, Sun Young Park, Yoko Nakano, Greg Mueller, and Andrew Ko. How designers design and program interactive behaviors. In $V L / H C C$, pages 177-184, 2008.

[10] Brian P. Bailey, Joseph A. Konstan, and John V. Carlis. Demais: designing multimedia applications with interactive storyboards. In ACM Multimedia, pages 241250, 2001.

[11] Mark W. Newman and James A. L. Sitemaps, storyboards, and specifications: A sketch of web site design practice. In 'DIS 2000 Designing Interactive Systems, pages 263-274. ACM Press, 2000.

[12] Jeffrey Nichols and Tessa Lau. Mobilization by demonstration: using traces to re-author existing web sites. In IUI '08: Proceedings of the 13th international conference on Intelligent user interfaces, pages 149-158, New York, NY, USA, 2008. ACM. 
[13] Yang Li and James A. Landay. Activity-based prototyping of ubicomp applications for long-lived, everyday human activities. In Proceedings of the Conference on Human Factors in Computing Systems, CHI 2008, pages 1303-1312, 2008.

[14] Corrie van der Lelie. The value of storyboards in the product design process. Personal Ubiquitous Computing, 10(2-3):159-162, 2006.

[15] Ron Sova and Deborah Hinderer Sova. Storyboards: a dynamic storytelling tool. Technical report, Sova Consulting Group, Tec-Ed Inc., 2006.

[16] Jan Meskens, Jo Vermeulen, Kris Luyten, and Karin Coninx. Gummy for multiplatform user interface designs: Shape me, multiply me, fix me, use me. In Proceedings of the working conference on Advanced Visual Interfaces, AVI 2008, New York, NY, USA, 2008. ACM. 